\title{
Reliability Improvement of RF Identification of Shadowed People in Building Corridors Using Space Diversity
}

\author{
Milan SVANDA ${ }^{1}$, Milan KVICERA ${ }^{2}$, Milan POLIVKA ${ }^{1}$ \\ ${ }^{1}$ Dept. of Electromagnetic Field, Czech Technical University in Prague, Technicka 2, 166 27, Prague 6, Czech Republic \\ ${ }^{2}$ Active Safety Product Line, VALEO Autoklimatizace k.s. Prague, Czech Republic \\ svandmil@fel.cvut.cz, milan.kvicera@valeo.com,polivka@fel.cvut.cz
}

Submitted April 12, 2018 / Accepted July 5, 2018

\begin{abstract}
This paper deals with the investigation of space diversity techniques for elimination of signal fading and consequently extension of coverage area in applications of $R F$ identification of shadowed people in a building corridor. Several measurement scenarios were evaluated. They comprised up to four wearable tags placed at different positions on a human body with one or two reader antennas, including shadowing by a small crowd of people. It was shown that the achieved corresponding SIMO mode diversity gain accounted for ca. $1 \mathrm{~dB}$ at the overall improvement in the trace coverage amounted to ca. $5 \%$. On the other hand, the MIMO mode diversity configuration brought an improvement of up to $10 \%$ and enabled to cover more than $50 \%$ of the identification area, including the scenario where shadowing by a small crowd of people was present.
\end{abstract}

\section{Keywords}

Patch antenna, radiofrequency identification, space diversity

\section{Introduction}

To date, the RFID technology is commonly used in a wide range of areas such as identification of items in warehouses, presence systems in buildings, parking systems, check-ins, logistics, etc. [1]. In recent years, researchers have dealt with the development of RFID transponders for special applications, including identification of objects made of metallic or liquid materials [2-5] and identification of people using transponders situated in the vicinity or directly placed on human bodies [6-9]. On the contrary, [10], [11] deal with the tag diversity of bodyworn antennas in the case that the shadowing effect of identified people is omitted. Further, the sensing of electrical or non-electrical quantities performed using the RFID transponders represents an up-to-date topic as well [12-14].

For all the above-mentioned applications, a high reliability of identification has to be ensured. The identifica- tion of people, especially in buildings, represents the application whose power budget is highly affected by multipath effects and shadowing in the surrounding environment. The power budget is influenced by the RF power transmitted by the reader, gain of all antennas, free-space loss between the reader and tag antenna and additional losses specific to the interference of electromagnetic waves in the given conditions. These additional power losses should be evaluated and the power backup has to be reflected in system calculations. To a certain degree, they can be minimized by optimal system setting. In addition, space diversity techniques, investigated in this paper, can be used for further improvement of the coverage area.

The paper analyzes the influence of space diversity techniques employed in order to eliminate the signal fading and to extend read range in the RFID applications for identification of shadowed people, who primarily move down the corridor towards the reader gate. The sport events or scenarios similar to airport security checks are typical examples. We therefore evaluated a number of representative measurement scenarios, where up to four wearable tags were placed at different positions on a human body, and either one or two reader antennas were utilized. The scenarios also included several configurations of the tagged person shadowed by a small crowd of six people.

\section{Tag Antenna Design}

The coupled patch antennas developed recently by the authors [6] were designed at the operation frequency of $2 \mathrm{GHz}$ for the measurements presented in this paper. The proposed antenna structure is composed of two patches shorted at the outer edges and coupled by a central gap, to which a pair of perpendicular comb notches is placed symmetrically, as depicted in Fig. 1. The complex and real input impedance of the antenna can be achieved by changing the number, length and width of the comb-notches and intermediate strips [6]. Following the utilized measurement system described in Sec. 3.1, the antenna was designed to be matched to the real impedance of $50 \Omega$. 


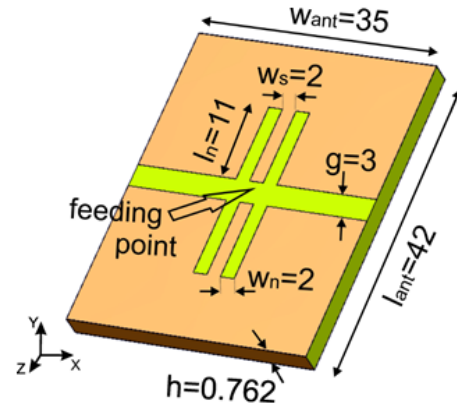

(a)

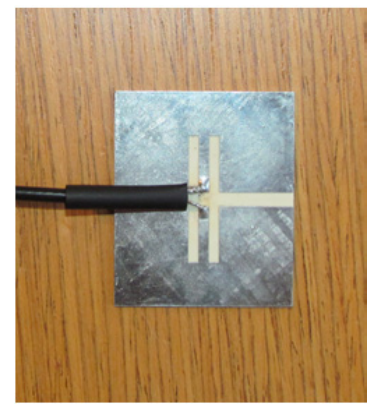

(b)
Fig. 1. Sketch (a) and photograph (b) of shorted coupled patch sensor antenna with comb-notches matched for $50 \Omega$ impedance (dimensions in $\mathrm{mm}$ ).

The total size of the antenna prototype is $42 \times 35 \times 0.762 \mathrm{~mm}^{3}$ (the relative size is $0.28 \times 0.23 \times 0.005 \lambda_{0}$ at $2 \mathrm{GHz}$ ). The measurement antenna sensor matched to $50 \Omega$ has two pairs of notches that are $11 \mathrm{~mm}$ in length. The other dimensions are shown in Fig. 1(a). The antenna was fabricated on the low-permittivity Rogers RO4350B woven-glass laminate with $\varepsilon_{\mathrm{r}}=3.48$, and loss tangent $\tan \delta=0.003$. The antenna feeding RG 174 flexible micro-coaxial cable was connected directly to the ends of the central pair of strips of the comb notches. Hence a differential feeding was formed. Although the antenna is a patch antenna operating over the ground plane, the symmetrization of the feeder was not implemented. The influence of non-perfect symmetrization on the radiation pattern tilt is assumed to be insignificant for application of the antenna in a complex environment (i.e. on the moving human body). Hence it does not decisively influence the propagation channel characteristics.

To verify its properties, the sensor antenna matched to $50 \Omega$ was first simulated by the IE3D method of moments simulator in free space and on a human phantom of the following dimensions: $120 \times 80 \times 15 \mathrm{~mm}^{3}$ in size with $\varepsilon_{\mathrm{r}} \sim 55$ and $\tan \delta \sim 0.5$. Such phantom enables direct contact of the rigid antenna, which is the worst case of the platform-tolerant antenna operation from the input impedance and radiation efficiency point of view. On a human body, more friendly configurations can be supposed (for instance the antenna is located on the clothing etc.). The comparison of simulated and measured reflection coefficients of the antenna in free space and on the human body is presented in Fig. 2. The reflection coefficient and the gain were measured in free space and also in the arrangement, where the antenna was attached to a human chest /phantom in an anechoic chamber. Very good agreement, and also a high immunity from the direct touch of a human body phantom, can be observed. Figure 3 shows the measured radiation patterns in both principal planes in free space and on the human body phantom.

\begin{tabular}{|l|c|}
\hline & Gain [dBi] \\
\hline Simulation - free space & 3.0 \\
\hline Simulation - human body phantom & 1.1 \\
\hline Measurement - free space & 2.9 \\
\hline Measurement - human body & 0.9 \\
\hline
\end{tabular}

Tab. 1. Gain of $50 \Omega$ coupled-patches antenna at frequency of $2 \mathrm{GHz}$.

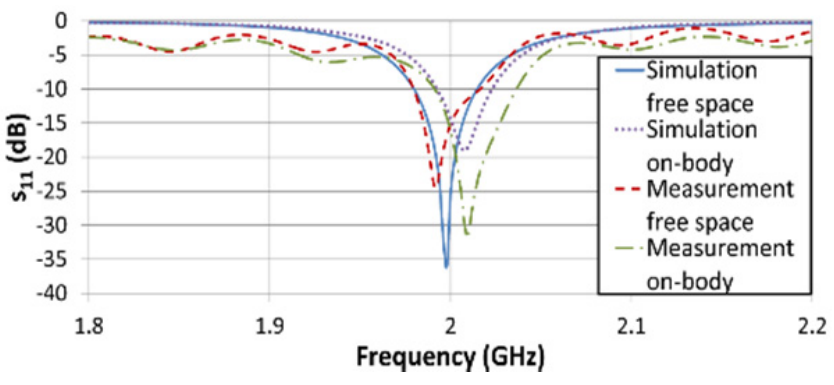

Fig. 2. Simulation and measurement of reflection coefficient of $50 \Omega$ shorted coupled patch antenna.

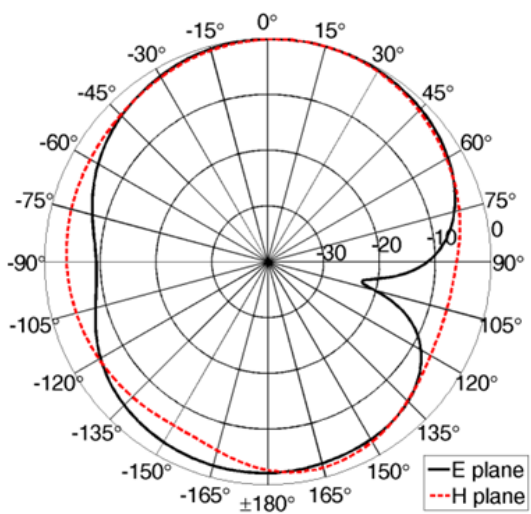

(a)

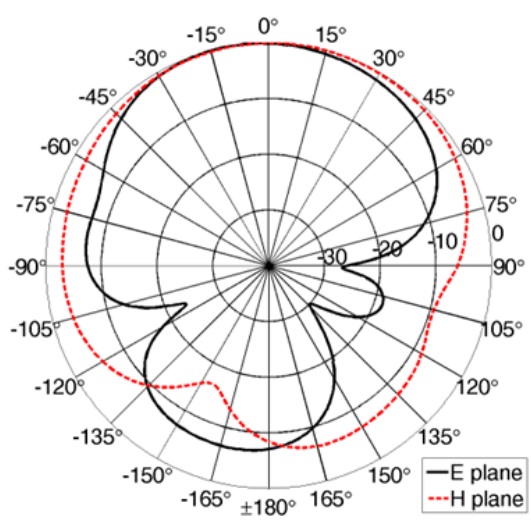

(b)

Fig. 3. Measured radiation patterns of coupled patch antenna with comb-slots in E plane and H plane: (a) In free space and (b) on human body phantom.

Table 1 provides the comparison of simulated and measured gain of the proposed antenna, where the results show a very good agreement.

\section{Building Corridor Measurements}

\subsection{Measurement System}

Depending on the selected measurement configuration, up to two transmit (Tx) rectangular patch antennas, denoted as TX1 and TX2 in Figs. 4 and 5, were utilized simultaneously as they were distinguished by their operating frequencies, 2001.06 MHz and 2000.86 MHz, respectively. 
To receive the continuous transmitted wave signals, a custom-made portable receiver with four parallel channels and a high sampling rate of $10 \mathrm{kHz}$ was used [15]. This receiver can be set so that all of its channels are tuned to the same frequency of $2001.06 \mathrm{MHz}$, denoted here as a single input multiple output (SIMO) mode. Alternatively, the first two channels are tuned to $2001.06 \mathrm{MHz}$ and the other two channels are tuned to $2000.86 \mathrm{MHz}$, referred to as a multiple input multiple output mode (MIMO). As a result, one Tx antenna and four receive $(\mathrm{Rx})$ antennas can be utilized at the same time in the SIMO mode. In contrast, in the MIMO mode, TX1 and TX2 are utilized concurrently and two Rx antennas are connected via two power splitters to the particular Rx inputs [15]. In each branch, the power splitters cause an additional loss of $3 \mathrm{~dB}$ in the system power budget.

To evaluate the relevancy of the selected scenarios, it is necessary to take into account the following parameters: $\mathrm{Rx}$ low noise floor of $-126 \mathrm{dBm}$ for a $12.5 \mathrm{kHz}$ bandwidth, common Tx rectangular patch antenna gain of about $9 \mathrm{dBi}$, $\mathrm{Rx}$ antenna gain from Tab. 1, available Tx output power of $-43 \mathrm{dBm}$, additional cable losses of approximately $5 \mathrm{~dB}$, and free space loss of about $65 \mathrm{~dB}$ for the maximum distance of $20 \mathrm{~m}$ between Tx and Rx. The system detected fades with amplitudes of at least $25 \mathrm{~dB}$ and $22 \mathrm{~dB}$ in the SIMO and MIMO mode, respectively, which makes it sufficient for the selected scenarios. The reconfigurable measurement system with well-suited parameters was previously verified by the authors during a number of measurements within different applications, see e.g. [15].

\subsection{Measurement Scenarios}

The measurements in both SIMO and MIMO mode configurations were performed in a four-meter wide building corridor with the ceiling height of $3.5 \mathrm{~m}$. The test person with Rx tag antennas was moving from a distance of $20 \mathrm{~m}$ towards Tx antennas at an approximately constant speed of $1 \mathrm{~m} / \mathrm{s}$; see Fig. 4.

The presented work is focused on investigation of actual scenarios, where separate RFID readers with a maximum output power (e.g. $1 \mathrm{~W}$ ) are supposed. This maximum output power is available for all outputs of the reader (or even for each reader if more readers are employed).

The measurements addressed three below-mentioned configurations of the tag, Tx antennas and several scenarios of shadowing caused by the presence of people.

- Configuration I: The system was operated in the SIMO mode and TX1 was located in the middle of the corridor, see Fig. 4b. Four tag antennas were situated on the chest, back and left and right shoulder of the test person, see RX1-RX4 in Fig. $4 a$.

- Configuration II: This configuration differs from the previous one only by the fact that TX2 placed at the edge of the corridor was utilized instead of TX1, see Figs. 4.

- Configuration III: The system was operated in the MIMO mode and both TX1 and TX2 were used at the same time. The two tag antennas (RX1, RX2) were located on the chest and left shoulder of the test person to achieve the best propagation conditions.

All above-described configurations were used for the following four measurement scenarios.

- Scenario I: The test person was moving alone in the middle of the corridor.

- Scenario II: The test person was moving alone along the right edge of the corridor.

- Scenario III: The test person was moving in the middle of the corridor, surrounded by a matrix of six people selected in a way that ensured that the propagation conditions were influenced in the most significant manner, see Fig. 4b.

- Scenario IV: The test person was moving along the right edge of the corridor, surrounded by a matrix of six people (identically to the Scenario III).

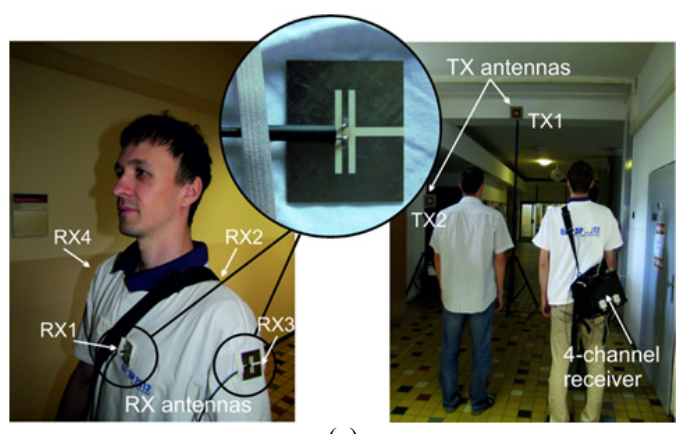

(a)

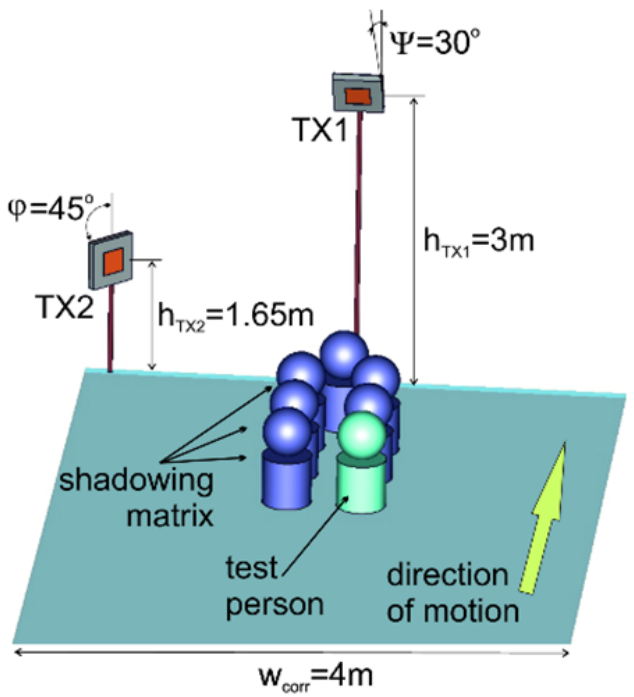

(b)

Fig. 4. Measurement setup of (a) Rx antennas on test person and Tx antennas in corridor, (b) geometry of Scenario III in $4 \mathrm{~m}$ wide building corridor (side walls and ceiling are removed). 


\section{Application of Space Diversity}

The selection combining algorithm [16] was utilized to evaluate the achieved spatial diversity. On condition that all $\mathrm{Rx}$ branches have identical noise powers, the resulting amplitude is simply the strongest one, which corresponds to the common RFID scenario where the tag with the response stronger than the chip sensitivity is being identified. Consequently, in SIMO mode with TX1 and $N$ Rx antennas, the resulting amplitude is considered to be as follows:

$$
r_{\mathrm{SIMO}}=\max \left(r_{1, \mathrm{TX} 1}, r_{2, \mathrm{TX} 1}, \ldots, r_{N, \mathrm{TX} 1}\right)
$$

with $r_{N \text { TX } 1}$ being the response amplitude from TX1 at the $N^{\text {th }}$ antenna in linear units (here $N=4$ ).

On the other hand, in the MIMO mode with two Tx and two Rx antennas, the overall strongest amplitude has to be selected in the following manner:

$$
r_{\mathrm{MIMO}}=\max \left(r_{1, \mathrm{TX} 1}, r_{1, \mathrm{TX} 2}, r_{2, \mathrm{TX} 1}, r_{2, \mathrm{TX} 2}\right) \text {. }
$$

It is also straightforward to extract the case of one $\mathrm{Rx}$ antenna and two Tx antennas from (2), which results in the multiple-input single-output (MISO) measurement mode. We may write then:

$$
r_{\mathrm{MISO}}=\max \left(r_{1, \mathrm{TX} 1}, r_{1, \mathrm{TX} 2}\right) .
$$

The diversity gain can be obtained for the individual measurement modes simply by subtracting the signal levels received by the particular $\mathrm{Rx}$ antenna from (1)-(3). For example, considering the improvement over the singleinput single-output (SISO) scenario with TX1 and RX1 only, the corresponding diversity gain in $\mathrm{dB}$ for each mode equals:

$$
\begin{aligned}
& G_{\mathrm{SIMO}}=10 \cdot \log _{10}\left(r_{\mathrm{SIMO}}\right)-10 \cdot \log _{10}\left(r_{1, \mathrm{TX} 1}\right), \\
& G_{\mathrm{MIMO}}=10 \cdot \log _{10}\left(r_{\mathrm{MIMO}}\right)-10 \cdot \log _{10}\left(r_{1, \mathrm{TX} 1}\right), \\
& G_{\mathrm{MISO}}=10 \cdot \log _{10}\left(r_{\mathrm{MISO}}\right)-10 \cdot \log _{10}\left(r_{1, \mathrm{TX} 1}\right) .
\end{aligned}
$$

Although TX1 and TX2 have the same output powers, they show/are operated at different frequencies, hence the powers received in particular Rx channels are not mutually influenced. By considering a realistic scenario where each tag reader constitutes a separate unit, the direct comparison between individual measurement modes is possible in (1)-(3).

Due to the multipath propagation and signal fading, the power level in the corridor shows an oscillatory character with power level minima and maxima. Subsequently, it is possible to identify several parts of each trace with signal coverage, i.e. where received signal level exceeds sensitivity of given transponder chip for the particular measurement mode. If the said individual trace lengths are summed up, denoted as $d_{\text {cover, }}$, and divided by the total trace length $d_{\text {total }}$, we obtain the trace coverage extent where reliable identification is achieved.

$$
\text { trace coverage extent }=\frac{d_{\text {cover }}}{d_{\text {total }}} \cdot 100 \% .
$$

The threshold levels represent the sensitivity thresholds of an RFID chip, i.e. the received signal level has to detect properly the signal from Tx. As the reverse direction from $\mathrm{Rx}$ towards $\mathrm{Tx}$ is less critical regarding the sensitivity of the system, we thus address the most critical part and automatically cover the correct identification of the tag.

Graphs of the trace coverage extent versus chip sensitivity are presented in Figs. 6-12, where the corresponding diversity gain can also be identified as it equals the change in the chip sensitivity when the spatial diversity mode is applied. It should be noted that as the measurement setup described in Sec. 3 represents the common RFID scenario with regard to the types of $\mathrm{Tx}$ and $\mathrm{Rx}$ antennas, the obtained experimental data were directly utilized in Figs. 6-12 after being corrected for the common RFID Tx output power of $1 \mathrm{~W}$. Commonly available chip sensitivities (at the time of the manuscript preparation) ranging from $-20 \mathrm{dBm}$ to $-5 \mathrm{dBm}$ have been applied here.

\subsection{SIMO Mode Measurements}

Figure 5 demonstrates the signal levels as measured in Configuration I within Scenario I. We clearly see that as the test person was moving towards $\mathrm{Tx}$, the selected chip sensitivity threshold of $-15 \mathrm{dBm}$ enabled to detect the tags continuously up from the distance of about $6 \mathrm{~m}$. However, it is not generally possible to directly evaluate the overall trace coverage extent of tag identification from such graphs as several peaks above the particular threshold appear at further distances as well. Thus, Figs. 6-12 address the particular measurement modes following (7).

In Fig. 6, the results for Configuration I under Scenario I are shown. If we aimed at a criterion stipulating the trace coverage extent above $50 \%$, e.g. $60 \%$, it was fulfilled only by utilizing the tag on the chest (RX1), considering the chip sensitivity around its limit of $-20 \mathrm{dBm}$. Employing the SIMO mode space diversity, such trace coverage extent could not be achieved without RX1. For instance, using both tags on the shoulders resulted only in the trace coverage extent below $40 \%$ for the limiting chip sensitivity of $-20 \mathrm{dBm}$; see the magenta dashed line with circular points in Fig. 6. Nevertheless, the SIMO mode with all Rx antennas improved the trace coverage extent by about $5 \%$ for any chip sensitivity, while providing a low diversity gain of about $1 \mathrm{~dB}$. Other SIMO mode configurations involving RX1 were always between the SISO results for RX1 and the SIMO mode case with all four antennas; see Figs. 6-12. They were therefore omitted to keep these figures wellarranged. The little effect of RX2 placed on the back of the test person is also obvious from Figs. 6-12, as it could have been expected.

When keeping Configuration I and addressing Scenario II, we see the trace coverage extent improvement for $\mathrm{RX} 3$ and RX4 caused by the better orientation of RX3 
radiation pattern towards TX1 and stronger reflections from the wall close to the test person, respectively (Fig. 7). Yet, as in the previous case, the requirement of at least $50 \%$ trace coverage extent could be fulfilled only in the case when RX1 was utilized, irrespective of the SIMO mode.

By employing TX2 at the edge of the corridor (Configuration II), we noticed a behavior similar to the one in the case of Configuration I, with an overall slight increase in trace coverage extent (Fig. 8). Following Fig. 4a, this phenomenon resulted from the better pointing of TX2. When the test person was moving along the corridor's edge, the resulting characteristics in Fig. 9 remained very similar to those in Fig. 7.

As the overall differences between the results obtained in the case of Configurations I and II and Scenarios I and II were very small, measurements for the case of shadowing by the matrix of six people (Fig. 4a), are going to be demonstrated only for Configuration I and Scenario III. In comparison to Figs. 6-9, Figure 10 then depicts a substantial decrease in the influence of RX1 on the resulting identification trace coverage extent. Moreover, as can be seen in Fig. 4a, RX3 on the left shoulder was oriented along the isle within the shadowing matrix, whereas RX4 was more influenced by the presence of people in front of it, resulting in the better trace coverage extent levels of RX3. Again, the SIMO mode diversity improvement in the case of all $\mathrm{Rx}$ antennas was about 5\% for any chip sensitivity with a low diversity gain (ca. $1 \mathrm{~dB}$ ).

To sum up, by comparing Scenarios I and III for Configuration I (Figs. 6 and 10), we come to the conclusion that the $60 \%$ trace coverage extent criterion could not be met in Fig. 10. Moreover, due to shadowing, the extent of trace coverage slightly diminished by about $5 \%$ for RX3 and RX4, which further weakened their influence within the applied SIMO mode diversity.

\subsection{MIMO Mode Measurements}

In Figs. 11 and 12, we show representative results for the MIMO mode, namely Scenarios I and III in Configura-

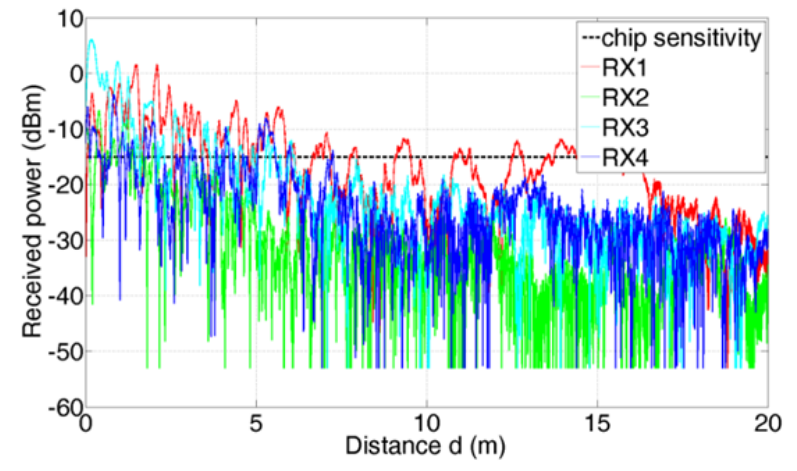

Fig. 5. Example of measured power levels versus distance $d$ from TX1, Configuration I, Scenario I. Horizontal line at $15 \mathrm{dBm}$ represents chip sensitivity. Trace coverage extent is the relative extent of the trace (compared to the total trace length) where the received power is higher than the chip sensitivity.

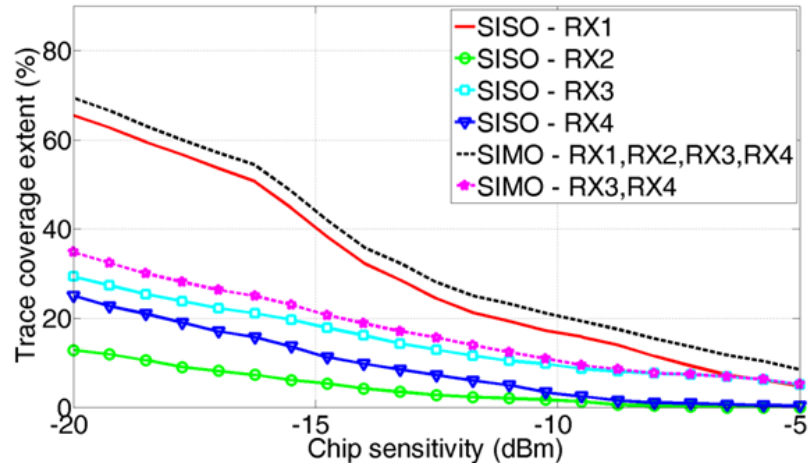

Fig. 6. Trace coverage extent for reliable identification versus common RFID chip sensitivities, Configuration I, Scenario I.

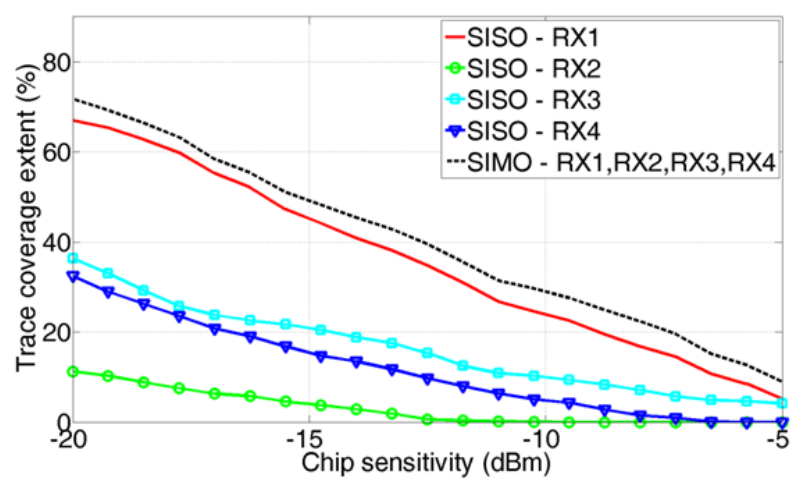

Fig. 7. Trace coverage extent for reliable identification versus common RFID chip sensitivities, Configuration I, Scenario II.

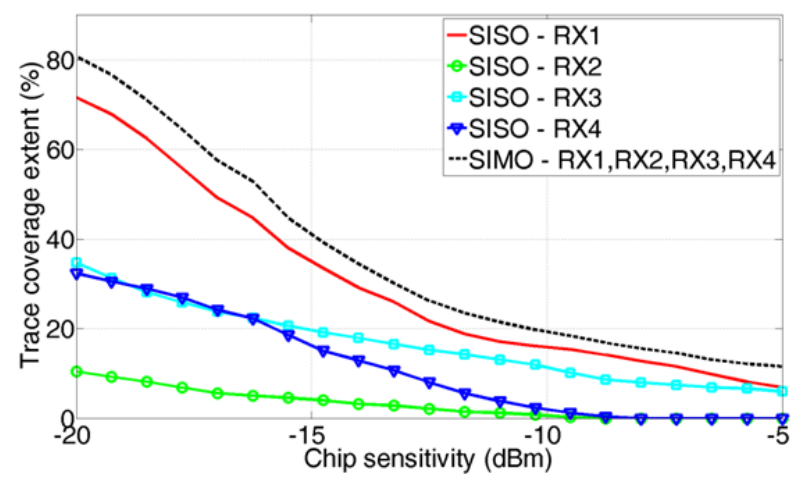

Fig. 8. Trace coverage extent for reliable identification versus common RFID chip sensitivities, Configuration II, Scenario I.

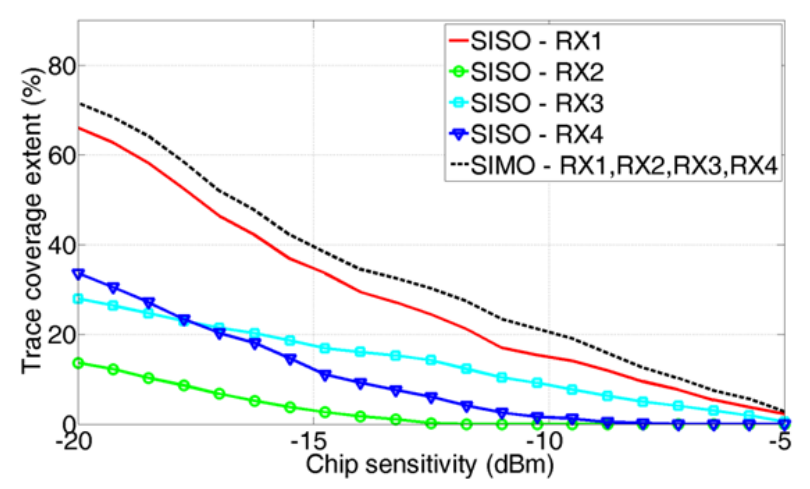

Fig. 9. Trace coverage extent for reliable identification versus common RFID chip sensitivities, Configuration II, Scenario II. 


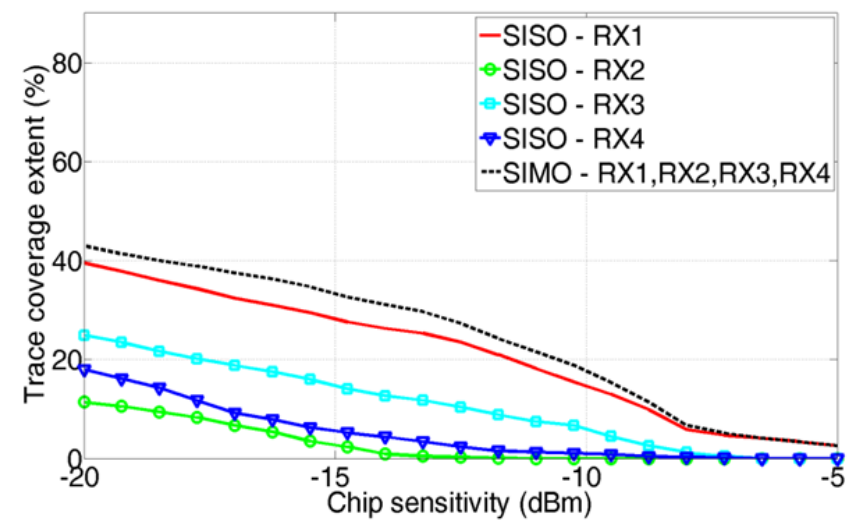

Fig. 10. Trace coverage extent for reliable identification versus common RFID chip sensitivities, Configuration I, Scenario III.

tion III. Taking into consideration the results from the previous Sec. 4.1, both TX1 and TX2 were utilized simultaneously and tag antennas were selected to be located on the test person's chest and left shoulder (RX1 and RX3) to address the best propagation conditions, i.e. within which the maximum identification area coverage with minimum number of antennas was attained.

Figure 11 indicates the results for Scenario I, where the test person was moving in the middle of the corridor without any shadowing. In comparison to the SIMO mode case in Fig. 6, we mainly notice an increase in the SISO TX1-RX1 channel, while in contrast to Fig. 8, there is a slight drop in the SISO TX2-RX1 channel. As this is attributed to the same channels and thus should be identical, it proves that the repeatability of measurements may be rather limited by the repeatability of the actual test person's route and the tag placement steadiness, influenced by the test person's movement.

Taking these limitations into account, we present results for the case of shadowing by the matrix of people in Fig. 12, which can be compared to the results in Fig. 10. In this case, the repeatability of shadowing conditions may apply as well. However, it is obvious that the identification trace coverage extent levels above 50\% were hardly reached and the resulting MIMO mode diversity improvement equals, again, merely 5\%. From this perspective, assuming

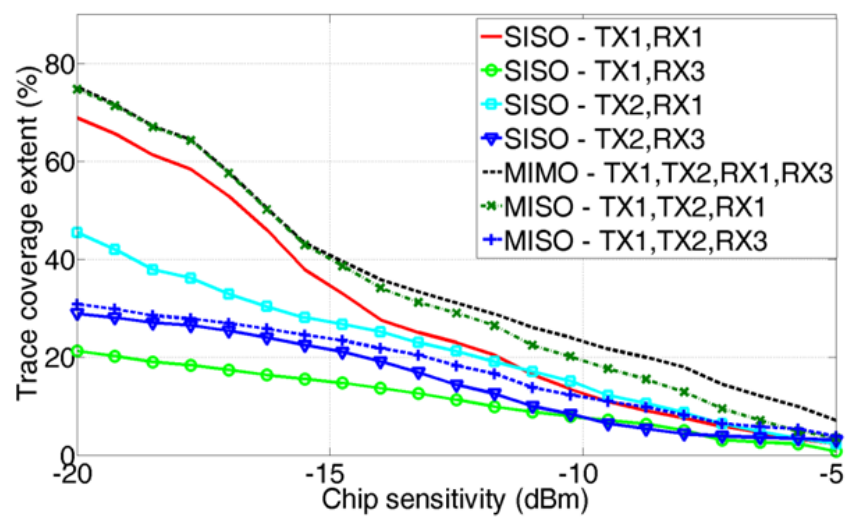

Fig. 11. Trace coverage extent for reliable identification versus common RFID chip sensitivities, Configuration III, Scenario I.

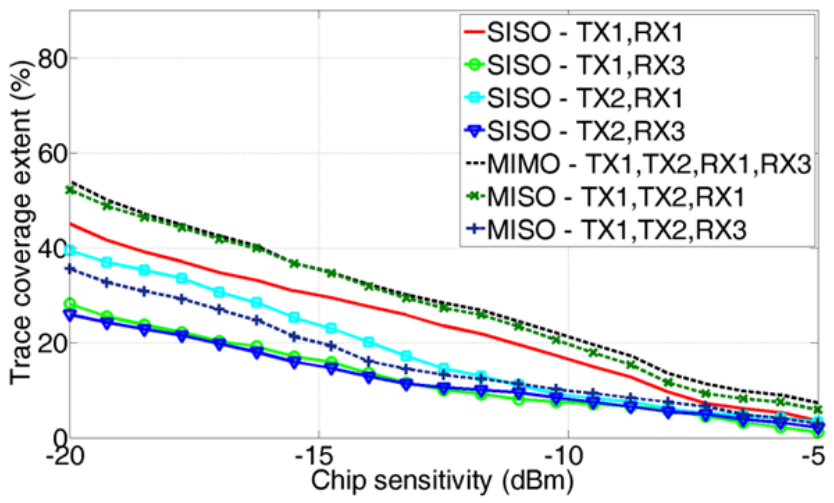

Fig. 12. Trace coverage extent for reliable identification versus common RFID chip sensitivities, Configuration III, Scenario III.

that RX1 is utilized, the MIMO mode configuration does not introduce any significant advantage over the SIMO mode diversity case.

\subsection{Discussion}

In regard to the SISO mode results in Sec. 4.1, it can be deduced for the considered scenarios that the best placement of the tag antenna is on the person's chest. Yet the overall trace coverage extent percentage of the tag identification ranged approximately from $40 \%$ to $70 \%$, depending on the chip sensitivity and type of scenario. It should be pointed out that in case a power margin is required, the trace coverage extent proportionally decreases.

This can be improved, to a certain level, by applying the space diversity. Although it has turned out that another antenna on the person's shoulder is a suitable solution, such placement is less comfortable for the user in case of rigid badge type tag antenna as it may not be completely stable for any kind of test person's movement. Indeed, it is necessary to consider the utilized antenna type. This can be avoided, for instance, by using tag antennas designed on flexible substrates.

On the contrary, a tag antenna situated on the back brings only a small improvement to trace coverage extent when a person approaches the face to the identification gate. Despite the obviously negligible effect of RX2 placed on the back of the test person, RX2 could enable the identification of the test person in the case that this person completely turns back from Tx or leaves the corridor. However, as stated in the introduction, such cases are not major concerns for the selected corridor scenarios, where the wearer walks towards the reader antenna. Generally speaking, if the person turns around, additional tags placed on other parts of his/her body increase their significance for identification process.

It was shown that the corresponding SIMO mode diversity gain was merely about $1 \mathrm{~dB}$, whereas the improvement in the trace coverage extent was worth some $5 \%$. In the MIMO mode with Rx antennas on the chest and left shoulder, the improvement ranged from $5 \%$ to $10 \%$ even in the case that shadowing of the test person occurred, which enabled to cover more than $50 \%$ of the identification area. 
Yet given the fact that the tag antenna on the chest is utilized, the MIMO mode configuration introduces only a small advantage over the SIMO mode diversity within the studied scenarios and considered range of chip sensitivities. If we strictly take into account exclusively the costs, more diversified portfolio of components on the tag-side seems to represent a better solution. However, as mentioned above, in certain cases (e.g. identification of people by the badge type tag antennas) every additional tag antenna reduces the user comfort. Consequently, the acceptable tradeoff between the increased number of used identification antennas and user comfort might be found in specific applications where multiple fully-wearable antennas (e.g. sewned) are inherent parts of working clothes, i.e. in case of firefighters, rescuers, etc.

\section{Conclusion}

The paper is focused on the space diversity techniques aimed at eliminating signal fading in RF identification systems utilized for identification of shadowed people primarily moving through the corridor towards a reader gate. It was shown that for the considered frequencies and scenarios within the common SISO mode, the tag antenna situated on the person's chest provides a considerably better signal coverage of the identification area than antennas placed on the test person's shoulders or back.

By applying the space diversity, either SIMO or MIMO modes, it was possible to raise the trace coverage extent of tag identification by about 5\% (10\% in the best case) compared to the case when only one antenna placed on the chest was utilized. As a result, the configuration with two Tx antennas and one chest-fixed tag antenna seems to be a comfortable solution for the user. It is also suitable from the trace coverage extent point-of-view. The diversity can therefore be ensured on the reader part (two Tx antennas), while the tag part consisting of a single tag antenna situated on the person's chest is represented by an identification badge. However, it should be noted that once the test person turns back, additional tags placed on other parts of his/her body increase their significance for successful identification, in other words in case of both approaching forehead and leaving back turned two Rx antennas are necessary for reliable identification.

\section{Acknowledgments}

This work has received support from the Czech Science Foundation project No. 17-02760S (experiments) and by COST project No. LD14122 (simulations).

\section{References}

[1] FINKENZELLER, K. RFID Handbook: Fundamentals and Applications in Contactless Smart Cards and Identification. John Wiley \& Sons, 2005. DOI:10.1002/9780470665121
[2] SVANDA, M., POLIVKA, M. Horizontal five-arm folded dipole over metal screening plane for UHF RFID of dielectric objects. Microwave and Optical Technology Letters, 2010, vol. 52, no. 10, p. 2291-2294. DOI: $10.1002 /$ mop. 25475

[3] ALI BABAR, A., BJORNINEN, T., BHAGAVATI, V. A., et al. Small and flexible metal mountable passive UHF RFID tag on high-dielectric polymer-ceramic composite substrate. IEEE Antennas and Wireless Propagation Letters, 2012, vol. 11, p. 1319-1322. DOI: 10.1109/lawp.2012.2227291

[4] LIN, K-H., CHEN, S-L., MITTRA, R. A looped-bowtie RFID tag antenna design for metallic objects. IEEE Transactions on Antennas and Propagation, 2013, vol. 61, no. 2, p. 499-505. DOI: 10.1109/tap.2012.2224082

[5] MO, L., QIN, CH. Planar UHF RFID tag antenna with open stub feed for metallic objects. IEEE Transactions on Antennas and Propagation, 2010, vol. 58, no. 9, p. 3037-3043. DOI: 10.1109/tap.2010.2052570

[6] SVANDA, M., POLIVKA, M. Matching technique for an on-body low-profile coupled-patches UHF RFID tag and for sensor antennas. IEEE Transactions on Antennas and Propagation, 2015, vol. 63, no. 5, p. 2295-2301. DOI: 10.1109/tap.2010.2052570

[7] SVANDA, M., POLIVKA, M., HUDEC, P. Novel low-profile foam dielectric over the shoulder antenna based on coupled patches technique. Microwave and Optical Technology Letters, 2013, vol. 55, no. 3, p. 593-597. DOI: 10.1002/mop.27389

[8] SANZ-IZQUIERDO, B., HUANG, F., BATCHELOR, J. C. Covert dual-band wearable button antenna. Electronic Letters, 2006, vol. 42, no. 12, p. 668-670. DOI: 10.1049/el:20060482

[9] MANZARI, S., PETTINARI, S., MARROCCO, G. Miniaturised wearable UHF-RFID tag with tuning capability. Electronic Letters, 2012, vol. 48, no. 21, p. 1325-1326. DOI: 10.1049/el.2012.2813

[10] MANZARI, S., OCCHIUZZI, C., MARROCCO, G. Feasibility of body-centric systems using passive textile RFID tags. IEEE $\mathrm{An}$ tennas and Propagation Magazine, 2012, vol. 54, no. 4, p. 49-62. DOI: 10.1109/map.2012.6309156

[11] OYEKA, D., BATCHELOR, J. C., ZIAI, A. M., et al. Tag diversity of inkjet printed body-worn RFID integrated medical sticking plasters for wireless monitoring. IET Healthcare Technology Letters, 2016, vol. 3, no. 3, p. 1-6. DOI: 10.1049/htl.2016.0024

[12] OCCHIUZZI, C., CAIZZONE, S., MARROCCO, G. Passive UHF RFID antennas for sensing applications: Principles, methods and classifications. IEEE Antennas and Propagation Magazine, 2013, vol. 55, no. 6, p. 14-34. DOI: 10.1109/map.2013.6781700

[13] CAIZZONE, S., DI GIAMPAOLO, E., MARROCCO, G. Constrained pole-zero synthesis of phase-oriented RFID sensor antennas. IEEE Transactions on Antennas and Propagation, 2016, vol. 64, no. 2, p. 496-503. DOI: 10.1109/tap.2015.2511788

[14] KRACEK, J., SVANDA, M., MAZANEK, M., MACHAC, J. Implantable semi-active UHF RFID tag with inductive wireless power transfer. IEEE Antennas and Wireless Propagation Letters, 2016, vol. 15, no. 1, p. 1657-1660. DOI: 10.1109/lawp.2016.2520663

[15] KVICERA, M., PECHAC, P. Seasonal variations of polarization diversity gain in a vegetated area considering high elevation angles and a nomadic user. International Journal of Antennas and Propagation, 2015, Article ID 194626, p. 1-9. DOI: $10.1155 / 2015 / 194626$

[16] SAUNDERS, S. R. Antennas and Propagation for Wireless Communication Systems. $2^{\text {nd }}$ ed. Wiley, 2007, p. 407. ISBN-13: 978-0470848791

\section{About the Authors ...}

Milan SVANDA was born in Prague, Czech Republic, in 1982. He received the M.S. and Ph.D. degrees in Radio 
Electronics from the Czech Technical University in Prague (CTU), in 2007 and 2011, respectively. He currently works as research scientist at CTU in Prague. He is an author or co-author of more than 40 papers published in international journals or conference proceedings and co-author of five patents. His main research activities are focused on antennas operating in the close vicinity of the human body, lowprofile and wearable antennas. His research interest is in UHF radiofrequency identification (RFID) of people and also in developing TAG antennas and sensors for special applications.

Milan KVICERA was born in Mestec Kralove, Czech Republic. He received the M.Sc. degree and the Ph.D. degree in Radio Electronics from the Czech Technical University in Prague, Czech Republic, in 2008 and 2012, respectively. He was a PostDoc from 2012 to 2015 and a researcher from 2015 to 2017, both at CTU in Prague. In 2017, he joined VALEO Autoklimatizace k.s. He has pub- lished more than 20 papers in international journals or conference proceedings and his research interests are focused on radio wave propagation especially regarding scattering from vegetation, satellite communications and automotive radar.

Milan POLIVKA was born in Prague, Czech Republic, in 1971. He received the M.S. and Ph.D. degrees in Radio Electronics from the Czech Technical University in Prague, Czech Republic, in 1996 and 2003, respectively. He is currently an Associate Professor and serves as a Vice-dean for doctoral study and science with the CTU in Prague, Faculty of Electrical Engineering. He is an author or coauthor of more than 100 papers published in international journals or conference proceedings and co-author of eight patents. His current research interests include antennas, microwave technique, metamaterials, chipless radiofrequency identification and applied electromagnetism. 